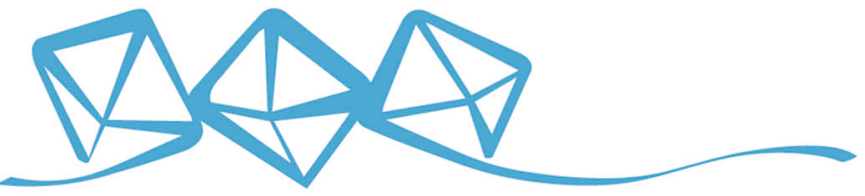 COMMUNICATIONS MATERIALS
}

ARTICLE

https://doi.org/10.1038/s43246-020-0018-1

OPEN

\section{Absence of superconductivity in bulk $\mathrm{Nd}_{1-x} \mathrm{Sr}_{x} \mathrm{NiO}_{2}$}

Qing $\mathrm{Li}^{1,2}$, Chengping $\mathrm{He}^{1,2}$, Jin $\mathrm{Si}^{1}$, Xiyu Zhu ${ }^{1 凶}$, Yue Zhang ${ }^{1} \&$ Hai-Hu Wen ${ }^{1 凶}$

Superconductivity at $9-15 \mathrm{~K}$ was recently discovered in $\mathrm{Nd}_{0.8} \mathrm{Sr}_{0.2} \mathrm{NiO}_{2}$ films. Since the $\mathrm{Ni}^{1+}$ ionic state in $\mathrm{NdNiO}_{2}$ may have the same $3 d^{9}$ outer-shell electronic orbital as in cuprate superconductors, it is interesting to know whether superconductivity has a similar mechanism in these two systems. Here we synthesize bulk samples of $\mathrm{Nd}_{1-x} \mathrm{Sr}_{x} \mathrm{NiO}_{2}(x=0$, $0.2,0.4)$ with inhomogeneous $\mathrm{Sr}$ distribution and Ni deficiency. Resistivity measurements show insulating behavior without the presence of superconductivity, different to the previously reported films. Although applying pressure up to about 50.2 GPa significantly suppresses the insulating behavior, superconductivity remains absent. The magnetization behavior exhibits a Curie-Weiss law with a paramagnetic moment of about $2 \mu_{\mathrm{B}} / \mathrm{f}$.u. Since the lattice constants derived from our diffraction data are very close to the previously reported superconducting $\mathrm{Nd}_{0.8} \mathrm{Sr}_{0.2} \mathrm{NiO}_{2}$ films, we suggest that superconductivity in that system may have arisen from interface or stress-related effects, or nickel deficiency in our bulk samples that might prevent the emergence of superconductivity.

\footnotetext{
${ }^{1}$ National Laboratory of Solid State Microstructures and Department of Physics, Center for Superconducting Physics and Materials, Collaborative Innovation Center for Advanced Microstructures, Nanjing University, Nanjing 210093, China. ${ }^{2}$ These authors contributed equally: Qing Li, Chengping He.

凶email: zhuxiyu@nju.edu.cn; hhwen@nju.edu.cn
} 
S ince the discovery of high critical temperature superconductivity (HTS) in cuprates in $1986^{1}$, there are plenty of experimental and theoretical studies to explore the intrinsic mechanism for superconductivity ${ }^{2-5}$. There is a general agreement that the parent compound of cuprates like $\mathrm{La}_{2} \mathrm{CuO}_{4}$ is a Mott insulator with a charge transfer gap and long-range antiferromagnetic (AF) order ${ }^{6}$. With chemical doping, the long-range AF order will be suppressed at a hole doping level $(p \approx 0.02)$ and $d$-wave superconductivity emerges at a higher doping level $(p \geq$ $0.05)^{7-9}$. After the efforts more than three decades, some common features have been observed, but the intrinsic pairing mechanism of HTS remains unresolved yet. These include twodimensional electronic structure and coexistence with an AF order or spin fluctuations, all these also occur in most ironbased $^{10}$ and heavy fermion superconductors ${ }^{11}$. Moreover, in cuprates, it is also important that the spin $S=1 / 2$ magnetic moment from $3 d^{9}$ electrons forms the basic structure of the AF order. One intuitive way to explore the pairing mechanism of cuprates is to find additional high- $T_{\mathrm{C}}$ superconductors with different transition metals but similar crystal and electronic structure ${ }^{12,13}$. The infinite-layer materials $R_{\mathrm{NiO}}(R=\mathrm{La}, \mathrm{Nd})$ are one of the ideal systems to simulate cuprates. First, the $R \mathrm{NiO}_{2}$ compounds have the same crystal structure $(P 4 / \mathrm{mmm})$ as $\mathrm{CaCuO}_{2}$, which is a parent compound of high- $T_{\mathrm{C}}$ cuprates and can reach a high- $T_{\mathrm{C}}$ of about $110 \mathrm{~K}$ by hole doping ${ }^{14}$. Second, the $\mathrm{Ni}^{1+}\left(3 d^{9}\right)$ oxidation state in $R \mathrm{NiO}_{2}$ is very similar to $3 d^{9}$ configuration of $\mathrm{Cu}^{2+}$ in cuprates. Thus, many theoretical and experimental efforts have been put forward to investigate the $R \mathrm{NiO}_{2}$ as a promising candidate of cuprate-like Ni-based superconductor ${ }^{12,15-17}$.

Recently, superconductivity was observed at $9-15 \mathrm{~K}$ in the strontium doped infinite-layer nickelate thin films of $\mathrm{Nd}_{0.8} \mathrm{Sr}_{0.2} \mathrm{NiO}_{2}$ deposited on the $\mathrm{SrTiO}_{3}$ substrate ${ }^{18}$. This work has stimulated enormous interests, and plenty of theoretical works have been carried out ${ }^{19-31}$. Among them, Zhang et al. propose the parent compound of $\mathrm{Nd}_{0.8} \mathrm{Sr}_{0.2} \mathrm{NiO}_{2}$ as a self-doped Mott insulator ${ }^{26}$. This work suggests that the low-density $N d-5 d$ conduction electrons couple with the localized Ni-3d electrons, which suppresses the long-range AF order and forms Kondo spin singlets at low temperatures. While, Botana and Norman argue that a large ratio of longer-range hopping to near-neighbor hopping is conducive for superconductivity both in cuprates and nickelates ${ }^{20}$. And Ryee et al. demonstrate that magnetic two-dimensionality induced by hole doping is the key factor for superconducting $\mathrm{Nd}_{1-x} \mathrm{Sr}_{x} \mathrm{NiO}_{2}{ }^{25}$. Bernardini et al. ${ }^{31}$ propose a possible difference between cuprates and nickelates based on the computed London penetration depth, and suggest that the latter does not follow the Uemura plot which holds well in underdoped cuprates. Hirayama et al. ${ }^{30}$ compare the electronic structure of cuprates and $\mathrm{NdNiO}_{2}$, and conclude that the $\mathrm{Nd}$ layer also forms Fermi pockets. And they also propose some other promising compounds analogues to high- $T_{\mathrm{C}}$ cuprates. Several other groups also studied the dominant pairing instability in the framework of $t-J$ model $^{23,27}$. They proposed that superconductivity in nickelates has a $d$-wave symmetry, which is analogous to cuprates. However, since the report of discovering superconductivity in $\mathrm{Nd}_{0.8} \mathrm{Sr}_{0.2} \mathrm{NiO}_{2}{ }^{18}$ thin films, no other experimental works have been reported up to now.

In this paper, we report the successful synthesis and physical properties of bulk $\mathrm{Nd}_{1-x} \mathrm{Sr}_{x} \mathrm{NiO}_{2}(x=0,0.2,0.4)$ samples. By using a three-step method, we prepare bulk Sr-doped $\mathrm{NdNiO}_{2}$ polycrystalline samples successfully with the similar doped composition of $\mathrm{Sr}$ as in the reported films. The structural and composition analyses reveal the formation of bulk $\mathrm{Nd}_{1-x} \mathrm{Sr}_{x} \mathrm{NiO}_{2}$ phase which at this moment has low crystallinity, nickel deficiency, and inhomogeneous strontium distribution. The low crystallinity may be a common feature for the low-temperature topotactic reduction method, which can also be seen in the bulk form $\mathrm{NdNiO}_{2}$ samples ${ }^{32}$ and the superconducting $\mathrm{Nd}_{0.8} \mathrm{Sr}_{0.2} \mathrm{NiO}_{2}{ }^{18}$ thin film. Magnetization measurements of the sample show a Curie-Weiss (C-W) like feature at high fields $(1$ and $3 \mathrm{~T})$. The resistivity measurements at ambient and high pressures exhibit insulating behavior without the presence of superconductivity. We think the contradictory results compared with that of the reported films ${ }^{18}$ may be attributed to the interface or stress effect in films. Furthermore, in our samples, we find appreciable deficiency (5-9\%) of $\mathrm{Ni}$ in the $\mathrm{NiO}_{2}$ planes. It would be interesting to know whether this feature occurs also in the reported films, which may lead to the absence of superconductivity in our bulk samples.

\section{Results}

Sample characterization. Figure 1a shows the schematic crystal structures and the transformation from the 113 to 112 phase through the low-temperature topotactic reduction method. Here $\mathrm{Nd} / \mathrm{Sr}, \mathrm{Ni}$ and oxygen atoms are represented by the orange/ purple, green and red spheres, respectively. To verify the formation of bulk $\mathrm{Nd}_{1-x} \mathrm{Sr}_{x} \mathrm{NiO}_{2}$ phase, we conduct the room temperature XRD measurements with $2 \theta$ from $10^{\circ}$ to $90^{\circ}$ and the Rietveld refinements ${ }^{33}$ of $\mathrm{Nd}_{1-x} \mathrm{Sr}_{x} \mathrm{NiO}_{2}(x=0.2,0.4)$ bulk samples. The results are shown in Fig. 1b, c. The XRD data of undoped $\mathrm{NdNiO}_{2}$ sample are shown in Supplementary Fig. 1. One can see that the Sr-doped samples have better crystallinity, which may be due to the fact that the valence of $\mathrm{Ni}$ in $\mathrm{Nd}_{1-x} \mathrm{Sr}_{\mathrm{x}} \mathrm{NiO}_{2}(x=0.2,0.4)$ is closer to $2+$, and it is known that the $\mathrm{Ni}^{2+}$ state is much more stable than $\mathrm{Ni}^{1+}$. All the samples
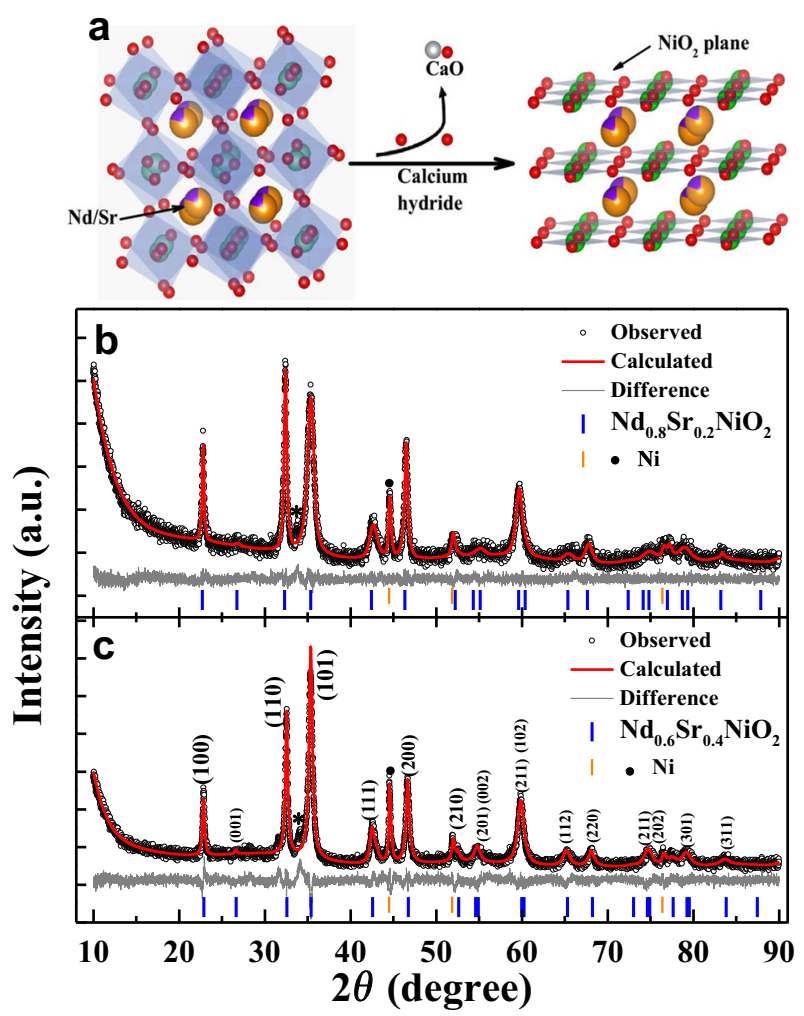

Fig. 1 Crystal structure and Rietveld refinement analyses. a The schematic structure and transformation from perovskite $\mathrm{Nd}_{1-x} \mathrm{Sr}_{x} \mathrm{NiO}_{3}$ to infinite-layer $\mathrm{Nd}_{1-x} \mathrm{Sr}_{x} \mathrm{NiO}_{2}$ by low-temperature reduction process with $\mathrm{CaH}_{2}$. b, $\mathbf{c}$ Powder X-ray diffraction patterns of $\mathrm{Nd}_{0.8} \mathrm{Sr}_{0.2} \mathrm{NiO}_{2}$ and $\mathrm{Nd}_{0.6} \mathrm{Sr}_{0.4} \mathrm{NiO}_{2}$ (circles) and Rietveld fitting curves (red lines) to the data. A small impurity peak around 33 degree marked with asterisk $\left(^{*}\right)$ in both samples may come from the unreacted $(\mathrm{Nd}, \mathrm{Sr})_{2} \mathrm{NiO}_{4}$ phase. The Miller indices of $\mathrm{Nd}_{0.6} \mathrm{Sr}_{0.4} \mathrm{NiO}_{2}$ sample are given in $\mathrm{C}$. 
contain mainly the infinite-layer 112 phase and some amount of extra $\mathrm{Ni}$ appears as disconnected segregations. Some peaks of $\mathrm{Nd}_{1-x} \mathrm{Sr}_{x} \mathrm{NiO}_{2}$ seem to be broader than others or have unexpected strong intensities, which may be induced by the disorder in these reflection planes and anisotropic particle size in layered materials. And the low crystallinity is a common feature for the samples fabricated by the low-temperature topotactic reduction method $^{32}$. From the indices of those peaks as shown in Fig. 1c, it is obvious that the diffraction peaks of the class $\langle h k 0\rangle$ are sharper. And the broader peaks are from the class where $l$ has a nonzero value. It means that the $\mathrm{NiO}_{2}$ plane may be disordered, which is corresponding to the result of the nickel deficiency in present samples as inferred from the EDS measurement. The crystallographic data obtained from the Rietveld refinement profiles are shown in Table 1 and Supplementary Table 1. The lattice constant of $c$-axis increases with increasing doping contents. The lattice parameters of our bulk sample $(\mathrm{x}=0.2)$ are $a=$ $3.91 \AA, c=3.33 \AA$, which are in good agreement with the previous reports on the reported $\mathrm{Nd}_{0.8} \mathrm{Sr}_{0.2} \mathrm{NiO}_{2}$ thin film ${ }^{18}$.

Figure $2 \mathrm{a}, \mathrm{b}$ display the scanning electron microscope (SEM) images of $\mathrm{Nd}_{1-x} \mathrm{Sr}_{x} \mathrm{NiO}_{2}(x=0.2,0.4)$ samples. As we can see, the grains could be clearly separated into two major morphologies, which are characterized by the argenteous crystals (dominant phase) and dark gray areas. The argenteous grains are interconnected, while the dark gray ones are well separated with each other. In order to have a deeper insight of the difference between the two different grains, energy dispersive X-ray spectroscopy (EDS) is used to analyze the element concentration of all three samples. In each sample, we measure more than 20 spots randomly (marked by the red crosses in images of Fig. 2a, b and Supplementary Fig. 1). The results are shown in Fig. 2c-f. As there is uncertainty for the composition of carbon and oxygen, here we only study the contents of $\mathrm{Nd}, \mathrm{Sr}$, and $\mathrm{Ni}$. Noting that the starting ratio of $\mathrm{Nd} / \mathrm{Sr} / \mathrm{Ni}$ in $\mathrm{Nd}_{1-x} \mathrm{Sr}_{x} \mathrm{NiO}_{2}$ is stoichiometric when we fabricate the sample. The typical EDS patterns of different samples are given in Fig. $2 c$, $d$, we find that the argenteous grains have a composition of $\mathrm{Nd}_{1-x} \mathrm{Sr}_{x} \mathrm{Ni}_{y} \mathrm{O}_{2}$, while the dark gray grains show a composition of $\mathrm{Ni}$ (see Supplementary Fig. 2 and Supplementary Note 1). Two typical features can be identified for the $\mathrm{Nd}_{1-x} \mathrm{Sr}_{x} \mathrm{Ni}_{y} \mathrm{O}_{2}$ : (1) The ratio of $\mathrm{Ni}$ : $(\mathrm{Nd}+\mathrm{Sr})$ is close to 1 , although an appreciable deficiency of $\mathrm{Ni}$ can be seen from the EDS data. Figure 2e shows the mean value of Ni occupancy normalized by the summed concentration of Nd and Sr. The deficiency of Ni should be in the scale of 5-9\% in all three samples $(x=0,0.2,0.4)$. The vacancies in nickel sites may cause the distortion of $\mathrm{NiO}_{2}$ plane and hence result in the shrinkage of the lattice constants of $a$ and $b$. And the disorder in those reflection planes may induce the peak broadening of XRD. As shown in Supplementary Table 1, the concentration of nickel metal extracted from our XRD data is about $15 \mathrm{wt} \%$ for $x=$ 0.2 sample and $17 \mathrm{wt} \%$ for $x=0.4$ sample, which is larger than the observed nickel deficiency in EDS measurement (about 5-9\%).

Table 1 Lattice parameters obtained from the Rietveld refinements.

\begin{tabular}{|c|c|c|c|c|}
\hline $\mathrm{Nd}_{0.8} \mathrm{Sr}_{0.2} \mathrm{NiO}_{2}$ & $\mathrm{P} 4 / \mathrm{mmm}$ & $51.01(6)$ & $3.9138(9)$ & $3.3303(8)$ \\
\hline $\mathrm{Nd}_{0.8} \mathrm{Sr}_{0.2} \mathrm{NiO}_{2}$ thin films ${ }^{18}$ & $\mathrm{P} 4 / \mathrm{mmm}$ & $51.06-51.67$ & 3.91 & $3.34-3.38$ \\
\hline
\end{tabular}

Lattice parameters obtained from the Rietveld refinements of $\mathrm{Nd}_{1-x} \mathrm{Sr}_{x} \mathrm{NiO}_{2}(x=0,0.2,0.4)$ at room temperature and a comparation with superconducting $\mathrm{Nd}_{0.8} \mathrm{Sr}_{0.2} \mathrm{NiO}_{2}$ thin film ${ }^{18}$.
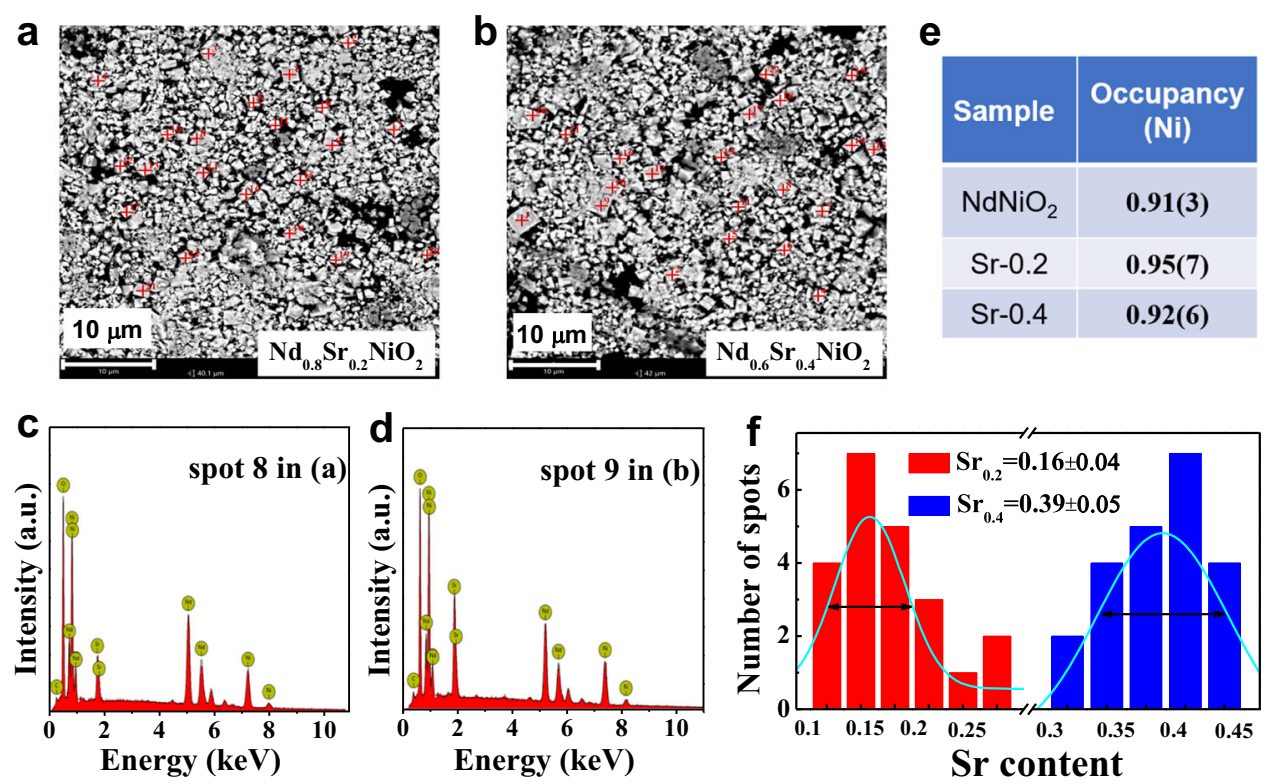

Fig. 2 Scanning electron micrograph images and energy dispersive spectroscopy analyses. a, b SEM images of $\mathrm{Nd}_{0.8} \mathrm{Sr}_{0.2} \mathrm{NiO}_{2}$ and $\mathrm{Nd}_{0.6} \mathrm{Sr}_{0.4} \mathrm{NiO}_{2}$ samples. c, d The typical energy dispersive spectroscopy (EDS) of the spot 8 in (a) and spot 9 in (b), respectively. e The mean value of occupied ratio of nickel in $\mathrm{Nd}_{1-x} \mathrm{Sr}_{x} \mathrm{NiO}_{2}(x=0,0.2,0.4)$ samples. $\mathbf{f}$ The proportion distribution of $\mathrm{Sr}$ concentration by measuring EDS on different grains of the samples. $\mathrm{A}$ clear inhomogeneity of strontium content is observed. The strontium content can be addressed as $0.16 \pm 0.04$ for $x=0.2$ sample and $0.39 \pm 0.05$ for $x=$ 0.4 sample from the Gaussian fitting. 

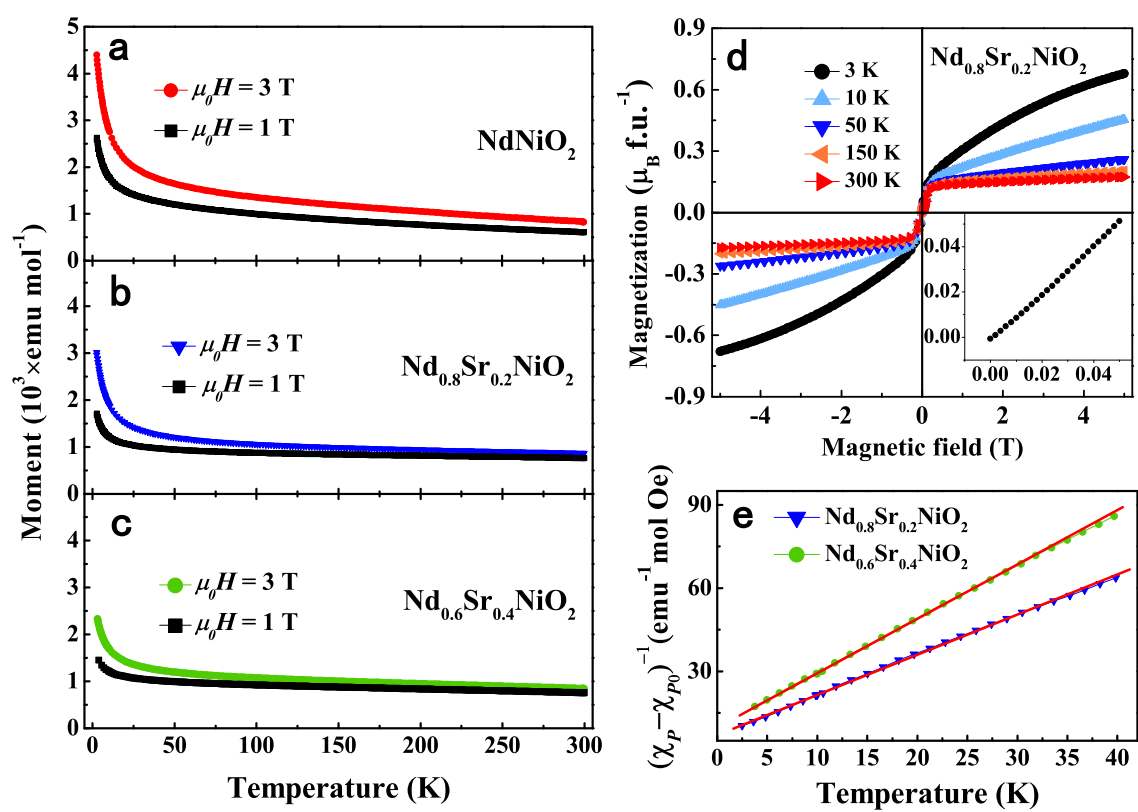

Fig. 3 Magnetic properties of $\mathbf{N d}_{\mathbf{1}-\mathbf{x}} \mathbf{S r}_{\mathbf{x}} \mathbf{N i O}_{\mathbf{2}}(\mathbf{x}=\mathbf{0}, \mathbf{0 . 2}, \mathbf{0 . 4})$ samples. a-c Temperature dependence of magnetic moment measured at the fields of 1 and $3 \mathrm{~T}$ for $\mathrm{NdNiO}_{2}, \mathrm{Nd}_{0.8} \mathrm{Sr}_{0.2} \mathrm{NiO}_{2}$ and $\mathrm{Nd}_{0.6} \mathrm{Sr}_{0.4} \mathrm{NiO}_{2}$ samples. d Magnetization hysteresis loops for $\mathrm{Nd}_{0.8} \mathrm{Sr}_{0.2} \mathrm{NiO}_{2}$ sample at different temperatures. Inset shows a linear relation between $M$ with $H$ at low field. e Temperature dependence of $\left(\chi_{P}-\chi_{P O}\right)^{-1}$ in the low-temperature region. The red line shows a linear relation of $\left(\chi_{P}-\chi_{P O}\right)^{-1}$ versus $T$.

The inconsistent content of nickel metal concentration in Rietveld refinement and the observed nickel deficiency in EDS measurement may be induced by the different crystallinity, different grain size as well as the preferred orientation between $\mathrm{Ni}$ cluster and grains of $\mathrm{Nd}_{1-x} \mathrm{Sr}_{x} \mathrm{NiO}_{2}$, which make the compositional ratio determined by XRD imprecise. (2) The doped $\mathrm{Sr}$ concentrations in $\mathrm{Nd}_{1-x} \mathrm{Sr}_{x} \mathrm{NiO}_{2}(x=0.2,0.4)$ samples are close to the nominal values with a clear inhomogeneity of strontium content among grains, which indicates the effective doping level of $\mathrm{Sr}$ in our samples. In Fig. 2f, we show the proportion distribution of $\mathrm{Sr}$ concentrations, more than half of the grains reach the nominal concentration of Sr. The strontium content for both samples show distributions which are fitted to Gaussian functions. Through the fitting, we find that the average strontium content can be expressed as $0.16 \pm 0.04$ for $x=0.2$ sample and $0.39 \pm 0.05$ for $x=0.4$ sample. Furthermore, for both $\mathrm{Nd}_{0.8} \mathrm{Sr}_{0.2} \mathrm{NiO}_{2}$ and $\mathrm{Nd}_{0.6} \mathrm{Sr}_{0.4} \mathrm{NiO}_{2}$ samples, the proportion of $\mathrm{Sr}$ and $\mathrm{Ni}$ in different grains all shows a clear distribution around the nominal value, but the concentrations of $\mathrm{Sr}$ and $\mathrm{Ni}$ on each measured point have no clear correlation (see Supplementary Fig. 3). As we know, the defects and inhomogeneity in transition metal oxides should play an important role in the electronic transport, which have been widely observed in cuprates. For example, by using an X-ray microdiffraction apparatus, scientists found that the superconducting transition temperature and charge-density-wave domain are highly affected by the inhomogeneous oxygen defect order ${ }^{34-36}$. And also in nickelates, by using the similar apparatus on a $\mathrm{NdNiO}_{3}$ film, the paradigm of nanoscale phase inhomogeneity has been extended to the correlated electron system with an AFM ground state ${ }^{37}$. Thus, we believe the electronic transport in $\mathrm{Nd}_{1-x} \mathrm{Sr}_{x} \mathrm{NiO}_{2}$ system may also be affected by the inhomogeneous distributions of $\mathrm{Sr}$ and $\mathrm{Ni}$ concentrations. Anyway, we believe our samples contain the right phase of $\mathrm{Nd}_{1-x} \mathrm{Sr}_{x} \mathrm{Ni}_{y} \mathrm{O}_{2}(x=0.2,0.4, y$ ranges from 0.91 to 0.95$)$. The sensitivity of the SQUID instrument should be sufficient to detect the superconducting signal if partial of these grains are superconductive.
Magnetic and Electrical transport properties. Figure $3 \mathrm{a}-\mathrm{c}$ shows the temperature dependence of magnetic moment $(M-T)$ curves at different external magnetic fields for $\mathrm{Nd}_{1-x} \mathrm{Sr}_{x} \mathrm{NiO}_{2}(x=0,0.2$, $0.4)$ samples. The magnetic moment of all samples at high fields $\left(\mu_{0} H=1 \mathrm{~T}\right.$ and $\left.3 \mathrm{~T}\right)$ increases with decreasing temperature in the whole temperature range, exhibiting approximately linear behavior at high temperature and a $\mathrm{C}-\mathrm{W}$ like enhancement at low temperature. It should be noted that the low field $\left(\mu_{0} H=1 \mathrm{mT}\right)$ magnetization of all three samples shows a drop of magnetization at about $5 \mathrm{~K}$, together with an obvious irreversibility between the ZFC and FC curves (see Supplementary Fig. 4). Such abnormal behavior at low field magnetization curve may indicate the presence of spin glassy state, probably due to magnetic impurities in compounds. To obtain a deeper understanding of magnetization behavior, we measure the magnetization hysteresis $(M-H)$ curves of the $\mathrm{Nd}_{0.8} \mathrm{Sr}_{0.2} \mathrm{NiO}_{2}$ sample at different temperatures and show the data in Fig. $3 \mathrm{~d}$. We can safely exclude the possibility that the anomalous drop of magnetization at about $5 \mathrm{~K}$ is caused by superconductivity, because no superconducting like $M-H$ curve is observed at $3 \mathrm{~K}$ (inset of Fig. 3d). The $M-H$ curves show the presence of paramagnetic background with a ferromagnetic component, and the paramagnetic component decreases as the temperature increases. We think the ferromagnetic component is originated from the segregated phase of $\mathrm{Ni}$. And the paramagnetic behavior is attributed to the $\mathrm{Nd}_{1-x} \mathrm{Sr}_{x} \mathrm{NiO}_{2}$ phase. We have tried to fit the high field $M-T$ curves in low-temperature region by the $\mathrm{C}-\mathrm{W}$ law $\chi=\chi_{0}+\frac{C}{T+T_{\theta}}$. The fitting results are given in Fig. $3 e$, as shown by the red solid line, indicating a good quality of $\mathrm{C}-\mathrm{W}$ fitting. Through the $\mathrm{C}-\mathrm{W}$ fitting, we derive the effective magnetic moments $\mu_{\text {eff }}$ of $\mathrm{Nd}_{0.8} \mathrm{Sr}_{0.2} \mathrm{NiO}_{2}$ and $\mathrm{Nd}_{0.6} \mathrm{Sr}_{0.4} \mathrm{NiO}_{2}$, which are about $2.32 \mu_{\mathrm{B}} /$ f.u. and $2.03 \mu_{\mathrm{B}} /$ f.u., respectively. It should be noted that, before doing the $\mathrm{C}-\mathrm{W}$ fitting, we need to remove the ferromagnetic background of nickel from the $M-T$ curves by subtracting the curves of $1 \mathrm{~T}$ from those of $3 \mathrm{~T}$. This is valid since the magnetization of Ni gets already saturated at the magnetic fields of $1 \mathrm{~T}$ and $3 \mathrm{~T}$, as shown in Supplementary Fig. 5. The subtracted paramagnetic magnetic 

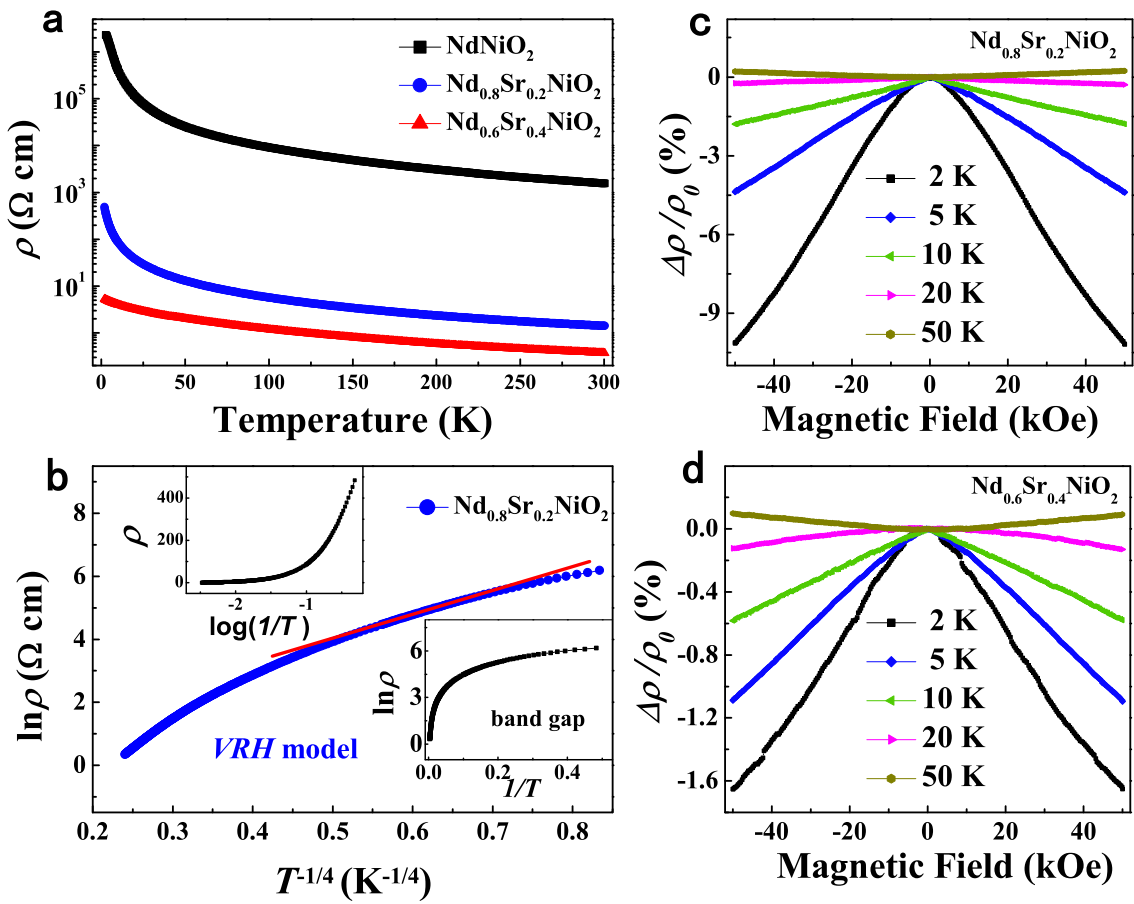

Fig. 4 Temperature dependent resistivity and magnetoresistance at ambient pressure. a Temperature dependence of resistivity for $\mathrm{Nd}_{1-x} \mathrm{Sr}_{x} \mathrm{NiO}_{2}(x=$ $0,0.2,0.4)$ samples. $\mathbf{b}$ Temperature dependence of resistivity in $\ln \rho$ versus $T^{-1 / 4}$ for $\mathrm{Nd}_{0.8} \mathrm{Sr}_{0.2} \mathrm{NiO}_{2}$. The top inset shows the curve of $\rho$ versus log $(1 / T)$ and the bottom inset shows the curve of $\ln \rho$ versus $1 / T$ (band gap model) for $\mathrm{Nd}_{0.8} \mathrm{Sr}_{0.2} \mathrm{NiO}_{2}$. c, $\mathbf{d}$ Field dependence of magnetoresistance $\Delta \rho / \rho_{0}$ at different temperatures for $\mathrm{Nd}_{0.8} \mathrm{Sr}_{0.2} \mathrm{NiO}_{2}$ and $\mathrm{Nd}_{0.6} \mathrm{Sr}_{0.4} \mathrm{NiO}_{2}$ samples. Both samples exhibit a reversal from negative magnetoresistance to positive magnetoresistance above $20 \mathrm{~K}$.

values $\chi_{P}=\left(M_{3 \mathrm{~T}}-M_{1 \mathrm{~T}}\right) / \Delta H$ is thus taken as the pure signal from the paramagnetic term. More details are given in Supplementary Note 2.

Electrical transport measurements are carried out at ambient pressure in the temperature range from 2 to $300 \mathrm{~K}$. Figure $4 \mathrm{a}$ shows the comparison of the temperature dependence of resistivity for $\mathrm{Nd}_{1-x} \mathrm{Sr}_{x} \mathrm{NiO}_{2}(x=0,0.2,0.4)$ samples. It can be clearly seen that the resistivity of the three samples all show insulating behaviors. The inhomogeneous distributions of $\mathrm{Sr}$ and $\mathrm{Ni}$ concentrations we discussed above may play an important role in electrical transport behavior, but it cannot affect the conclusion that superconductivity is absent in our samples. Assuming partial grains or some fraction of the grains show superconductivity below a certain temperature, the resistivity and magnetization should present a drop. To understand the underlying physics of the electrical transport behavior in present materials, in Fig. $4 \mathrm{~b}$ we present the $\rho(T)$ curve within the frame of variable range hopping (VRH) model ${ }^{38}$ described as $\rho=\rho_{0} \exp \left(T_{0} / T\right)^{-1 / 4}$, we can see that in a short period of temperature, it gives a linear relationship as highlighted by the red line, while the global fitting is not good. Also the band-gap model $(\ln \rho \propto 1 / T$, shown in the bottom-right inset) and resistivity versus $\log 1 / T$ (shown in the top-left inset) are plotted, but all failed to fit the data at ambient pressure, suggesting that the insulating is not originated from the band gap and should possess by itself some exotic scattering reasons. Figure $4 \mathrm{c}$, d shows the magnetic field dependent resistivity measured at different temperatures $(T=2,5,10,20$, $50 \mathrm{~K})$. A sizable negative magnetoresistance (MR) $\Delta \rho / \rho_{0}=$ $\left(\rho_{H}-\rho_{0}\right) / \rho_{0}$ (about $-10 \%$ at $5 \mathrm{~T}$ ) is observed at $2 \mathrm{~K}$. The negative MR at low temperature decreases with the increase of temperature, then the MR shows the crossover from a negative to a small positive MR behavior above $20 \mathrm{~K}$. The negative MR in correlated oxides may be attributed to various mechanisms such as hopping conduction, magnetic scattering, or de-localization effect. As for the positive MR, researchers have proposed that the exchange correlation in different hopping sites (VRH type conduction) could give rise to positive $\mathrm{MR}^{39,40}$. Owing to the nickel segregations in our samples, the origin of negative and positive MR observed in the present compounds is not clear and more works deserve to be done.

Resistivity under high pressure. As shown above, an obvious conclusion drawn from the $\rho(T)$ curves at ambient pressure is the insulating behavior. One can see that not only the magnitude of resistivity, but also the insulating features are strongly suppressed by doping more Sr. In order to induce a metallic or even a superconducting state, we also conduct high pressure electrical resistance measurement. The temperature dependence of resistivity at various pressures in $\mathrm{Nd}_{0.8} \mathrm{Sr}_{0.2} \mathrm{NiO}_{2}$ and $\mathrm{Nd}_{0.6} \mathrm{Sr}_{0.4} \mathrm{NiO}_{2}$ samples are shown in Fig. 5a and Supplementary Fig. 6. As discussed above, $\rho(T)$ curves of $\mathrm{Nd}_{1-x} \mathrm{Sr}_{x} \mathrm{NiO}_{2}$ exhibit an insulating behavior at ambient pressure. With application of pressure, the overall magnitude of the resistivity is continuously reduced. The insulating behavior is significantly weakened. The ratio $\rho_{2 \mathrm{~K}} / \rho_{300 \mathrm{~K}}$ of $\mathrm{Nd}_{0.8} \mathrm{Sr}_{0.2} \mathrm{NiO}_{2}$ sample is about 350 with the external pressure of $3.2 \mathrm{GPa}$. With increasing pressure, the magnitude of $\rho_{2 \mathrm{~K}} / \rho_{300 \mathrm{~K}}$ decreases progressively as shown in Fig. 5b. The improvement of conductivity under high pressures may be related to the redistribution of the Sr concentrations and modification of the nickel deficiency within grains. However, to prove this, one may need some microscopic analysis tools, such as synchrotron hard X-ray nanoimaging or small-angle scattering under high pressures, which have been used to reveal a novel micron-scaled ribbon phase in optimally doped $\mathrm{Bi}_{2} \mathrm{Sr}_{2} \mathrm{CaCu}_{2} \mathrm{O}_{8+\delta}{ }^{41}$. At the highest pressure of $50.2 \mathrm{GPa}$, the value of $\rho_{2 \mathrm{~K}} / \rho_{300 \mathrm{~K}}$ has dropped to 6 ; however the $\rho(T)$ curve still exhibits a semiconducting-like feature in whole temperature region. Then we try to fit the electrical transport behavior of $\mathrm{Nd}_{0.8} \mathrm{Sr}_{0.2} \mathrm{NiO}_{2}$ sample at the highest 

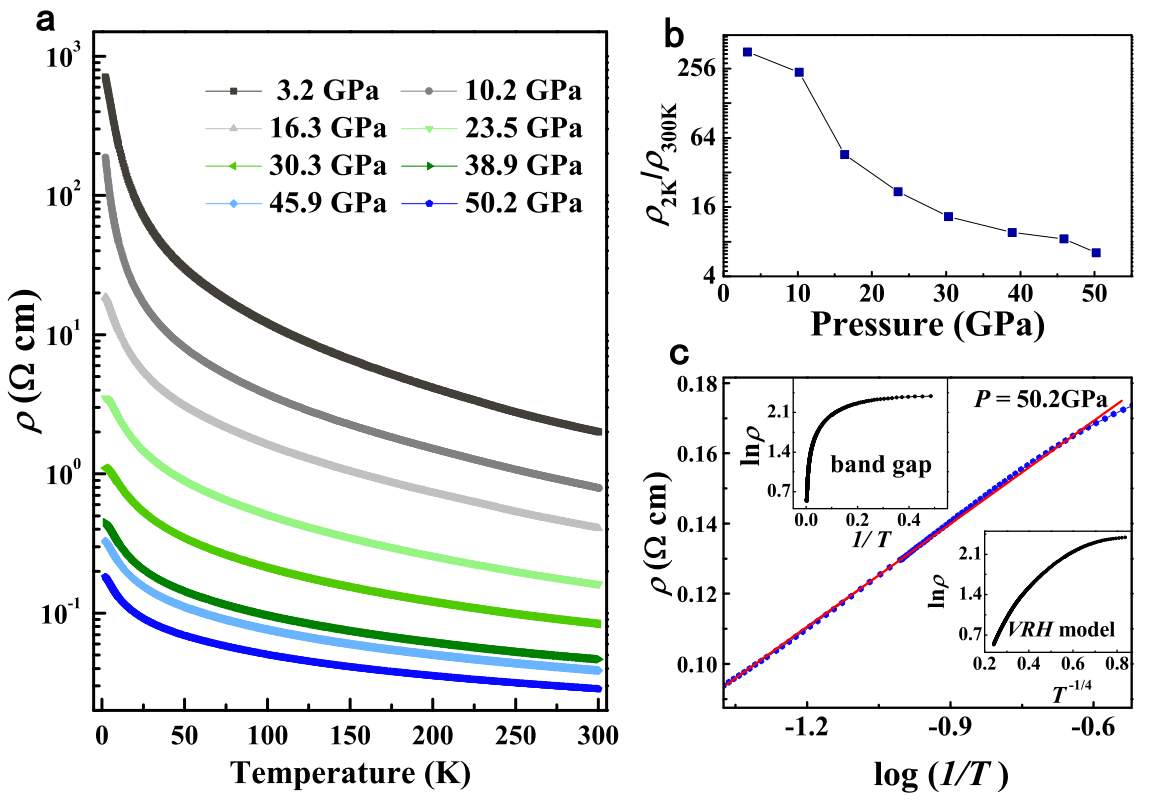

Fig. 5 Temperature dependent resistivity of $\mathbf{N d}_{\mathbf{0 . 8}} \mathrm{Sr}_{\mathbf{0 . 2}} \mathbf{N i O}_{\mathbf{2}}$ under pressures. a Temperature dependence of resistivity for $\mathrm{Nd}_{0.8} \mathrm{Sr}_{0.2} \mathrm{NiO}_{2}$ at various pressures. $\mathbf{b}$ The applied pressure dependence of $\rho_{2 \mathrm{~K}} / \rho_{300 \mathrm{~K}}$ for $\mathrm{Nd}_{0.8} \mathrm{Sr}_{0.2} \mathrm{NiO}_{2}$ sample derived from the $\rho-T$ curve at different pressures. c The $\rho$ versus In $(1 / T)$ curve for $\mathrm{Nd}_{0.8} \mathrm{Sr}_{0.2} \mathrm{NiO}_{2}$ in the temperature range from 3.5 to $23 \mathrm{~K}$ at the pressure of $50.2 \mathrm{GPa}$ together with the corresponding linear fit (red line). The top inset shows the curve of $\ln \rho$ versus $1 / T$, corresponding to band gap model. The bottom inset shows the curve of $\ln \rho$ versus $T^{-1 / 4}$, corresponding to the VRH model.

pressure and show the fitting in Fig. 5c. The fitting curves of band gap model (the top-left inset) and VRH model (the bottom-right inset) are given. The results show that both the band gap model and the VRH model fail to fit the data. Surprisingly, the $\rho$ versus $\log (1 / T)$ curve at the highest pressure $(P=50.2 \mathrm{GPa})$ becomes roughly linear, as highlighted by the red linear line in a temperature range between 3.5 and $23 \mathrm{~K}$. This strange $\rho \propto \log (1 / T)$ behavior has been reported in some correlated oxides such as underdoped and overdoped cuprates or vanadium oxide $\mathrm{V}_{2} \mathrm{Se}_{2} \mathrm{O}$, which may result from the electron correlation effect in correlated oxides with $3 d$ transition metals ${ }^{42-44}$. Meanwhile, the high pressure may change the distribution of $\mathrm{Sr}$ element and $\mathrm{Ni}$ deficiency within the grains, but due to the high diffusing barrier between different grains, we would not believe the high pressure will change the composition distribution of $\mathrm{Sr}$ and $\mathrm{Ni}$ among different grains. Thus the improvement of conductivity under pressure may be attributed to two factors. (1) The pressure can improve the electric conduction through optimizing the grain boundaries among different grains; (2) The pressure can modify the electronic band structure by making the redistribution of $\mathrm{Sr}$ and $\mathrm{Ni}$ deficiency within each grain, as well as the bandwidth through compressing the atomic lattice. These possibilities can be better resolved in future work.

\section{Discussions}

After a systematic analysis of the data, a main experimental finding in bulk infinite-layer nickelates $\mathrm{Nd}_{1-x} \mathrm{Sr}_{x} \mathrm{NiO}_{2}(x=0,0.2$, 0.4 ) is the insulating behavior, which is in contrast to the metallic state and the superconductivity below $9-15 \mathrm{~K}$ in $\mathrm{Nd}_{0.8} \mathrm{Sr}_{0.2} \mathrm{NiO}_{2}$ thin film ${ }^{18}$. It is certainly very important to know whether the insulating behavior in the bulk $\mathrm{Nd}_{1-x} \mathrm{Sr}_{x} \mathrm{NiO}_{2}$ is intrinsic. Based on the nice fitting to the XRD data and composition analysis on the grains of the samples, we can safely conclude that the main phase in the samples is definitely the $\mathrm{Nd}_{1-x} \mathrm{Sr}_{x} \mathrm{NiO}_{2}(x=0.2,0.4)$ with only some disconnected segregation clusters of Ni. From previous literatures, we find that nickel is a metal with the resistivity of about $7.2 \mathrm{~m} \Omega \mathrm{cm}$ at room temperature ${ }^{45}$, so it would be a metallic behavior if the segregated grains of nickel form a conductive network. However, from our SEM image, the segregated nickel grains are well separated with each other. The behavior of resistance both in ambient and high pressure indicates that the insulating behavior is robust in our samples. Thus, the existence of disconnected nickel regions in samples may only affect the absolute value of resistivity, but not gives rise to the intrinsic insulating behavior. In principle, high pressure can compress the cell volume and reduce the lattice parameters of compound, resulting in insulator-metal transitions and even superconductivity 46,47 . The clear suppression of insulating behavior in our high-pressure study may be induced by the improving of the grain boundaries, modification of the bands, or the weakening of the correlation effect, which leads to an enhanced effective density of states at the Fermi energy.

Since we do not find superconductivity in our bulk samples with the same structure and close lattice constants as the reported films ${ }^{18}$, we would like to suggest several reasons to interpret this discrepancy. Firstly, the superconductivity in $\mathrm{Nd}_{0.8} \mathrm{Sr}_{0.2} \mathrm{NiO}_{2}$ films may arise from the interface or the stress effect given by the substrates. In the interface region, both the electron band and the doping level would be strongly modified, which could lead to superconductivity. The second reason may be the appreciable $\mathrm{Ni}$ deficiency (5-9\%) in grains of $\mathrm{Nd}_{1-x} \mathrm{Sr}_{x} \mathrm{NiO}_{2}$ of our samples, which may cause stronger buckling of the $\mathrm{NiO}_{2}$ planes and lead to a strong localization or scattering of charges. If this is the case, we need to make samples without $\mathrm{Ni}$ deficiency. It would be interesting to know whether the reported films also have Ni deficiency.

To summarize, we have successfully synthesized bulk $\mathrm{Nd}_{1-x}$ $\mathrm{Sr}_{x} \mathrm{NiO}_{2}(x=0,0.2,0.4)$ samples and performed comprehensive studies of the physical properties. From structure and composition analyses by XRD and EDS, the tetragonal $\mathrm{Nd}_{1-x} \mathrm{Sr}_{x} \mathrm{NiO}_{2}$ $(x=0,0.2,0.4)$ phase is established and the strontium content is inhomogeneous and sufficient in most grains, but the $\mathrm{Ni}$ is deficient. The electrical transport and magnetization measurements reveal an insulating behavior and a $\mathrm{C}-\mathrm{W}$ like feature with a ferromagnetic background caused by nickel segregations. 
Electrical transport measurements under high pressure show that the insulating behavior can be effectively suppressed but superconductivity is still not observed.

\section{Methods}

Sample growth. To synthesize polycrystalline samples of $\mathrm{Nd}_{1-x} \mathrm{Sr}_{x} \mathrm{NiO}_{2}(x=0,0.2$, 0.4 ), we first prepare polycrystalline $\mathrm{Nd}_{2-2 x} \mathrm{Sr}_{2 x} \mathrm{NiO}_{4}(x=0,0.2,0.4)$ samples by the solid-state reaction of $\mathrm{Nd}_{2} \mathrm{O}_{3}, \mathrm{NiO}$, and $\mathrm{SrO}$ at $1200^{\circ} \mathrm{C}$ for $24 \mathrm{~h}$. And then we use precursor $\mathrm{Nd}_{2-2 x} \mathrm{Sr}_{2 x} \mathrm{NiO}_{4}, \mathrm{NiO}$, and $\mathrm{KClO}_{4}$ to synthesize the $\mathrm{Nd}_{1-x} \mathrm{Sr}_{x} \mathrm{NiO}_{3}(x=$ $0,0.2,0.4)$ with high pressure and high temperature. Here $\mathrm{KClO}_{4}$ is used as an excess oxygen source. Then the mixture is pressed into a pellet and sealed in a gold capsule. This procedure is done in a glove box with oxygen and water concentrations less than $0.1 \mathrm{ppm}$. The gold capsule is placed in a $B N$ capsule and heated up to $1000{ }^{\circ} \mathrm{C}$ and stayed for $2 \mathrm{~h}$ at this temperature under a pressure of $2 \mathrm{GPa}$. The resultant compound is the perovskite $\mathrm{Nd}_{1-x} \mathrm{Sr}_{x} \mathrm{NiO}_{3}$ phase which is verified by powder X-ray diffraction at room temperature (see Supplementary Fig. 7 and Supplementary Note 3). Next, the samples of $\mathrm{Nd}_{1-x} \mathrm{Sr}_{x} \mathrm{NiO}_{2}$ are obtained by reacting $\mathrm{Nd}_{1-x} \mathrm{Sr}_{x} \mathrm{NiO}_{3}(x=0,0.2,0.4)$ with $\mathrm{CaH}_{2}$, which is similar to the previous report ${ }^{48,49}$. The obtained $\mathrm{Nd}_{1-x} \mathrm{Sr}_{x} \mathrm{NiO}_{3}$ samples are washed by distilled water and then dried, ground with the $\mathrm{CaH}_{2}$ in a ratio of $1: 3$ and then pressed into pellets. The pellets are sealed in a quartz tube, followed by heating treatment at $280^{\circ} \mathrm{C}$ for $20 \mathrm{~h}$. Then the resultant pellets are crushed and washed with saturated $\mathrm{NH}_{4} \mathrm{Cl}$ in anhydrous ethanol to remove the residual $\mathrm{CaH}_{2}$ and the reacted byproduct $\mathrm{CaO}$, and then dried in an evacuated oven. The target sample of the 112 phase in powder form is obtained. For measuring resistivity, the powders are pressed into a pellet again, and sealed with untouched $\mathrm{CaH}_{2}$ powders in a quartz tube, followed by another heating treatment at $180^{\circ} \mathrm{C}$ for $10 \mathrm{~h}$

Physical properties measurements. The crystal structures of the prepared samples are determined by powder X-ray diffraction (XRD) (Bruker D8 Advance) using $\mathrm{Cu}-K \alpha$ radiation at room temperature with a scanning step of $0.01^{\circ}$ and $2 \theta$ from $10^{\circ}$ to $90^{\circ}$. The Rietveld refinements are done based on the TOPAS4.2 software ${ }^{32,50}$. The SEM photograph of the polycrystalline and the energy dispersive X-ray microanalysis spectrum are done by Phenom ProX (Phenom). The measurements are performed at an accelerating voltage of $15 \mathrm{kV}$. The DC magnetization is measured with a SQUID based on the vibrating sample technique (SQUID-VSM $7 T$, Quantum Design). The electrical resistances at ambient pressure are measured by the standard four-probe method using the physical property measurement system (PPMS 16T, Quantum Design). The high pressure resistivity measurements are performed by using the diamond avail cell (cryoDACPPMS, Almax easyLab) ${ }^{51}$.

\section{Data availability}

The data supporting the findings of this study are available from the corresponding author upon reasonable request.

Received: 25 November 2019; Accepted: 4 March 2020; Published online: 03 April 2020

\section{References}

1. Bednorz, J. G. \& Müller, K. A. Possible high $T_{\mathrm{c}}$ superconductivity in the Ba-La-Cu-O system. Z. Phys. B: Condens. Matter 64, 189-193 (1986).

2. Anderson, $\mathrm{P}$. W. The resonating valence bond state in $\mathrm{La}_{2} \mathrm{CuO}_{4}$ and superconductivity. Science 235, 1196-1198 (1987).

3. Tsuei, C. C. \& Kirtley, J. R. Pairing symmetry in cuprate superconductors. Rev. Mod. Phys. 72, 969-1016 (2000).

4. Lee, P. A., Nagaosa, N. \& Wen, X.-G. Doping a Mott insulator: physics of high-temperature superconductivity. Rev. Mod. Phys. 78, 17-85 (2006).

5. Keimer, B. et al. From quantum matter to high-temperature superconductivity in copper oxides. Nature 518, 179-186 (2015).

6. Vaknin, D. et al. Antiferromagnetism in $\mathrm{La}_{2} \mathrm{CuO}_{4-y}$. Phys. Rev. Lett. 58, 2802-2805 (1987).

7. Shen, Z.-X. et al. Anomalously Large Gap Anistropy in the $a-b$ Plane of $\mathrm{Bi}_{2} \mathrm{Sr}_{2} \mathrm{CaCu}_{2} \mathrm{O}_{8+\delta}$. Phys. Rev. Lett. 70, 1553-1556 (1993).

8. Wollman, D. A. et al. Experimental determination of the superconducting pairing state in YBCO from the phase coherence of YBCO-Pb dc SQUIDs. Phys. Rev. Lett. 71, 2134-2137 (1993).

9. Tsuei, C. C. et al. Pairing symmetry and flux quantization in a tricrystal superconducting ring of $\mathrm{YBa}_{2} \mathrm{Cu}_{3} \mathrm{O}_{7-\delta}$. Phys. Rev. Lett. 73, 593-596 (1994).

10. Kamihara, $\mathrm{Y}$. et al. Iron-based layered superconductor $\mathrm{La}\left[\mathrm{O}_{1-x} \mathrm{~F}_{x}\right] \mathrm{FeAs}(x=$ 0.05-0.12) with $T_{\mathrm{c}}=26 \mathrm{~K}$. J. Am. Chem. Soc. 130, 3296-3297 (2008).

11. Si, Q. \& Steglich, F. Heavy fermions and quantum phase transitions. Science 329, 1161-1166 (2010).

12. Chaloupka, J. \& Khaliullin, G. Orbital order and possible superconductivity in $\mathrm{LaNiO}_{3} / \mathrm{LaMO}_{3}$ superlattices. Phys. Rev. Lett. 100, 016404 (2008).
13. Zhang, J. et al. Large orbital polarization in a metallic square-planar nickelate Nature Phys. 13, 864-869 (2017).

14. Azuma, M. et al. Superconductivity at $110 \mathrm{~K}$ in the infinite-layer compound $\left(\mathrm{Sr}_{1-x} \mathrm{Ca}_{x}\right)_{1-y} \mathrm{CuO}_{2}$. Nature 356, 775-776 (1992).

15. Anisimov, V. I., Bukhvalov, D. \& Rice, T. M. Electronic structure of possible nickelate analogs to the cuprates. Phys. Rev. B 59, 7901-7906 (1999).

16. Boris, A. V. et al. Dimensionality control of electronic phase transitions in nickel-oxide superlattices. Science 332, 937-940 (2011).

17. Middey, S. et al. Physics of ultrathin films and heterostructures of rare-earth nickelates. Annu. Rev. Mater. Res. 46, 305-334 (2016).

18. Li, D. et al. Superconductivity in an infinite-layer nickelate. Nature 572, 624-627 (2019).

19. Hirsch, J. E. \& Marsiglio, F. Hole superconductivity in infinite-layer nickelates. Physica C: Supercond. Appl. 566, 1353534 (2019).

20. Botana, A. S. \& Norman, M. R. Similarities and differences between infinitelayer nickelates and cuprates and implications for superconductivity. Phys. Rev. X 10, 011024 (2020).

21. Sakakibara, H. et al. Model construction and a possibility of cuprate-like pairing in a new $d^{9}$ nickelate superconductor $(\mathrm{Nd}, \mathrm{Sr}) \mathrm{NiO}_{2}$. Preprint at https:// arxiv.org/abs/1909.00060 (2019).

22. Hepting, M. et al. Electronic structure of the parent compound of superconducting infinite-layer nickelates. Nat. Mater. (2020). https://doi.org/ 10.1038/s41563-019-0585-z.

23. Wu, X. et al. Robust $d_{x^{2}-y^{2}}$-wave superconductivity of infinite-layer nickelates. Phys. Rev. B 101, 060504 (2020).

24. Nomura, Y. et al. Formation of $2 \mathrm{D}$ single-component correlated electron system and band engineering in the nickelate superconductor $\mathrm{NdNiO}_{2}$. Phys. Rev. B 100, 205138 (2019).

25. Ryee, $\mathrm{S}$. et al. Induced magnetic two-dimensionality by hole doping in superconducting $\mathrm{Nd}_{1-x} \mathrm{Sr}_{x} \mathrm{NiO}_{2}$. Phys. Rev. B 101, 064513 (2020).

26. Zhang, G.-M., Yang, Y.-F. \& Zhang, F.-C. Self-doped Mott insulator for parent compounds of nickelate superconductors. Phys. Rev. B 101, 020501 (2020).

27. Zhang, Y.-H. \& Vishwanath, A. Type II $t-J$ model in superconducting nickelate $\mathrm{Nd}_{1-x} \mathrm{Sr}_{x} \mathrm{NiO}_{2}$. Preprint at https://arxiv.org/abs/1909.12865 (2019).

28. Gu, Y. et al. Hybridization and correlation effects in the electronic structure of infinite-layer nickelates. Preprint at https://arxiv.org/abs/1911.00814 (2019).

29. Hu, L.-H. \& Wu, C. Two-band model for magnetism and superconductivity in nickelates. Phys. Rev. Research 1, 032046 (2019).

30. Hirayama, M. et al. Materials design of dynamically stable $d^{9}$ layered nickelates. Phys. Rev. B 101, 075107 (2020).

31. Bernardini, F., Olevano, V. \& Cano, A. Magnetic penetration depth and $T_{C}$ in superconducting nickelates. Phys. Rev. Research 2, 013219 (2020).

32. Hayward, M. A. et al. Sodium hydride as a powerful reducing agent for topotactic oxide deintercalation: synthesis and characterization of the nickel(I) oxide $\mathrm{LaNiO}_{2}$. J. Am. Chem. Soc. 121, 8843-8854 (1999).

33. Rietveld, H. M. A profile refinement method for nuclear and magnetic structures. J. Appl. Cryst. 2, 65-71 (1969).

34. Fratini, M. et al. Scale-free structural organization of oxygen interstitials in $\mathrm{La}_{2} \mathrm{CuO}_{4+\mathrm{y}}$. Nature 466, 841-844 (2010).

35. Poccia, N. et al. Optimum inhomogeneity of local lattice distortions in $\mathrm{La}_{2} \mathrm{CuO}_{4+\mathrm{y}}$. Proc. Natl Acad. Sci. 109, 15685-15690 (2012).

36. Campi, G. et al. Inhomogeneity of charge-density-wave order and quenched disorder in a high- $\mathrm{T}_{\mathrm{c}}$ superconductor. Nature 525, 359-362 (2015).

37. Li, J. et al. Scale-invariant magnetic textures in the strongly correlated oxide $\mathrm{NdNiO}_{3}$. Nat. Commun. 10, 1-7 (2019).

38. Mott, N. F. \& Davis, E. A. Electronic Processes in Non-Crystalline Materials (Clarendon, Oxford, 1979).

39. Frydman, A. \& Ovadyahu, Z. Spin and quantum interference effects in hopping conductivity. Solid State Commun. 94, 745-749 (1995).

40. Vaknin, A. et al. High-field magnetoconductance in Anderson insulators. Phys. Rev. B 54, 13604-13610 (1996).

41. Zhang, J. et al. Evolution of a novel ribbon phase in optimally doped $\mathrm{Bi}_{2} \mathrm{Sr}_{2} \mathrm{CaCu}_{2} \mathrm{O}_{8+\delta}$ at high pressure and its implication to high- $\mathrm{T}_{\mathrm{C}}$ superconductivity. J. Phys. Chem. Lett. 9, 4182-4188 (2018).

42. Ando, Y. et al. Logarithmic divergence of both in-plane and out-of-plane normal-state resistivities of superconducting $\mathrm{La}_{2-\mathrm{x}} \mathrm{Sr}_{\mathrm{x}} \mathrm{CuO}_{4}$ in the zerotemperature limit. Phys. Rev. Lett. 75, 4662-4665 (1995).

43. Ono, S. et al. Metal-to-insulator crossover in the low-temperature normal state of $\mathrm{Bi}_{2} \mathrm{Sr}_{2-\mathrm{x}} \mathrm{La}_{\mathrm{x}} \mathrm{CuO}_{6+\delta}$. Phys. Rev. Lett. 85, 638-641 (2000).

44. Lin, H. et al. Structure and physical properties of $\mathrm{CsV}_{2} \mathrm{Se}_{2-x} \mathrm{O}$ and $\mathrm{V}_{2} \mathrm{Se}_{2} \mathrm{O}$. Phys. Rev. B 98, 075132 (2018).

45. Jordan, L. \& Swanger, W. H. The properties of pure nickel. Bur. Stand. J. Res. 5, 1291-1307 (1930).

46. Wang, Y. et al. Emergent superconductivity in an iron-based honeycomb lattice initiated by pressure-driven spin-crossover. Nat. Commun. 9, 1914 (2018).

47. Coak, M. J. et al. Isostructural Mott transition in $2 \mathrm{D}$ honeycomb antiferromagnet $\mathrm{V}_{0.9} \mathrm{PS}_{3}$. npj Quantum Mater. 38, (2019). 
48. Takamatsu, T. et al. Low-temperature synthesis of the infinite-layer compound $\mathrm{LaNiO}_{2}$ by soft-chemical techniques. Jpn. J. Appl. Phys. 49, 093101 (2010).

49. Hayward, M. A. \& Rosseinsky, M. J. Synthesis of the infinite layer Ni(I) phase $\mathrm{NdNiO}_{2+\mathrm{x}}$ by low temperature reduction of $\mathrm{NdNiO}_{3}$ with sodium hydride. Solid State Sci. 5, 839-850 (2003).

50. Cheary, R. W. \& Coelho, A. A fundamental parameters approach to x-ray line-profile fitting. J. Appl. Cryst. 25, 109-121 (1992).

51. Wang, E. et al. Pressure induced superconductivity in the compound ScZrCo. New J. Phys. 20, 073036 (2018).

\section{Acknowledgements}

This work was supported by the National Key R\&D Program of China (Grant No. 2016YFA0300401 and 2016YFA0401704), National Natural Science Foundation of China (Grant No. A0402/11534005 and A0402/11674164), and the Strategic Priority Research Program of Chinese Academy of Sciences (Grant No. XDB25000000).

\section{Author contributions}

The samples were grown by Q.L., C.P.H, Y.Z., and X.Y.Z. The physical properties measurements were conducted by Q.L. and J.S. The structure and composition analyses were done by Q.L., C.P.H. and X.Y.Z. H.-H.W., X.Y.Z., and Q.L. wrote the manuscript with the supplementary by others. All authors have discussed the results and the interpretations.

\section{Competing interests}

The authors declare no competing interests.

\section{Additional information}

Supplementary information is available for this paper at https://doi.org/10.1038/s43246 020-0018-1.

Correspondence and requests for materials should be addressed to X.Z. or H.-H.W.

Reprints and permission information is available at http://www.nature.com/reprints

Publisher's note Springer Nature remains neutral with regard to jurisdictional claims in published maps and institutional affiliations.

cc (i) Open Access This article is licensed under a Creative Commons Attribution 4.0 International License, which permits use, sharing, adaptation, distribution and reproduction in any medium or format, as long as you give appropriate credit to the original author(s) and the source, provide a link to the Creative Commons license, and indicate if changes were made. The images or other third party material in this article are included in the article's Creative Commons license, unless indicated otherwise in a credit line to the material. If material is not included in the article's Creative Commons license and your intended use is not permitted by statutory regulation or exceeds the permitted use, you will need to obtain permission directly from the copyright holder. To view a copy of this license, visit http://creativecommons.org/ licenses/by/4.0/.

(C) The Author(s) 2020 